\title{
L'autonomisation du « Parlement européen »
}

Interdépendance et différenciation des assemblées parlementaires supranationales (années 1950 - années 1970)

The Autonomization of the European Parliament: Interdependencies and differentiation of supranational parliamentary assemblies (1950s-1970s)

\section{Antonin Cohen}

\section{(2) OpenEdition}

\section{Journals}

Édition électronique

URL : http://journals.openedition.org/conflits/18288

DOI : $10.4000 /$ conflits. 18288

ISSN : $1777-5345$

Éditeur :

CCLS - Centre d'études sur les conflits lilberté et sécurité, L'Harmattan

Édition imprimée

Date de publication : 25 juin 2012

Pagination : 13-33

ISBN : 978-2-296-99408-9

ISSN : 1157-996X

Référence électronique

Antonin Cohen, «L'autonomisation du « Parlement européen » », Cultures \& Conflits [En ligne], 85-86 |

Printemps-Été 2012, mis en ligne le 25 juin 2013, consulté le 30 mars 2021. URL : http://

journals.openedition.org/conflits/18288; DOI : https://doi.org/10.4000/conflits.18288 


\title{
L'autonomisation du «Parlement européen»
}

\author{
Interdépendance et différenciation des assemblées \\ parlementaires supranationales (années 1950 - années 1970)
}

\section{Antonin COHEN}

Antonin Cohen est maître de conférences en science politique à l'Université Paris Ouest Nanterre La Défense et membre de l'Institut des sciences sociales du politique (CNRS, UMR 7220). Il a récemment publié De Vichy à la Communauté européenne (Paris, Presses Universitaires de France, 2012).

$\mathrm{L}$ es organisations intergouvernementales européennes restent trop souvent analysées de manière isolée les unes des autres, de sorte que la structure de cet archipel supranational se dérobe aux regards, même des plus avertis. Dans bien des travaux sur la construction européenne, le Conseil de l'Europe, l'Organisation du Traité de l'Atlantique nord, l'Union européenne et l'Union de l'Europe occidentale apparaissent en effet comme autant d'isolats. Au point que les enjeux militaires, économiques et politiques de la construction européenne semblent déconnectés les uns par rapport aux autres. Didier Bigo a pourtant bien montré que la formation des champs transnationaux ne pouvait être analysée en prenant simplement pour acquises les césures historiquement constituées entre les différentes organisations intergouvernementales ${ }^{1}$. Au contraire, même, cette différenciation doit être prise pour « objet » en tant que telle. La création de plusieurs organisations intergouvernementales distinctes doit en effet être comprise comme le résultat d'un processus hautement conflictuel, sans que ce résultat n'ait été anticipé, ni encore moins souhaité par aucun acteur étatique ou non étatique. Si ces frontières institutionnelles ont, par conséquent, une importance décisive dans la genèse de la construction européenne, elles ne peuvent être prises pour un découpage pertinent dans l'analyse du « champ du pouvoir européen 2 ». En cela, la démarche en termes

Mes remerciements s'adressent aux deux lecteurs anonymes de la première version de cet article.

1. Bigo D., Polices en réseaux. L'expérience européenne, Paris, Presses de la FNSP, 1996 ; voir aussi : Guiraudon V. (ed.), "Sociologie de l'Europe. Mobilisations, élites et configurations institutionnelles », Cultures E Conflits, 38-39, 2000.

2. Sur ce point : Cohen A., "Bourdieu Hits Brussels: The Genesis and Structure of the European 
de champs est profondément différente de la démarche en termes d'organisations, en ce qu'elle invite à restituer un espace de positions qui traverse les frontières instituées entre organisations et, partant, la multiplicité des positions institutionnelles ou informelles simultanément ou successivement occupées par les agents dans cet espace ${ }^{3}$.

\section{Une structure intergouvernementale centrale transversale}

Juridiquement séparées, les organisations intergouvernementales européennes sont, en effet, profondément enchevêtrées. Cette intrication transparaît dans la chronologie resserrée de création de ces différentes organisations, avec la signature du Traité de Bruxelles et de la Convention de Paris les 17 mars et 16 avril 1948, créant une Union occidentale (UO) et une Organisation européenne de coopération économique (OECE) ; avec la signature du Traité de Washington et du Statut de Londres les 4 avril et 5 mai 1949, fondant une Alliance Atlantique et un Conseil de l'Europe (CE) ; avec la signature du Traité de Paris et de la Convention d'Ottawa les 18 avril et 20 septembre 1951, instituant une véritable Organisation du Traité de l'Atlantique nord (OTAN) et une Communauté européenne du charbon et de l'acier (CECA); avec, enfin, dans une séquence qui résulte de la dynamique des succès et des échecs qui vont conjointement donner sa forme institutionnelle durable à « la » construction européenne, le Traité de Paris signé le 27 mai 1952 créant une Communauté européenne de défense (CED), dont la ratification est repoussée par l'Assemblée nationale française le 30 août 1954, les Accords de Paris du 23 octobre 1954 créant une Union de l'Europe occidentale (UEO) et les Traités de Rome signés le 25 mars 1957 instituant une Communauté économique européenne (CEE) et une Communauté européenne de l'énergie atomique (CEEA) ${ }^{4}$ [Tableau 1].

Ce rythme de négociation soutenu implique que les mêmes ministres sont bien souvent signataires de tous ces traités. Ainsi, le Belge Paul-Henri Spaak est resté en poste suffisamment longtemps pour signer le Traité de Bruxelles en 1948 et les Traités de Rome en 1957, et, en 1949, il peut signer d'un même mouvement de plume les traités créant à un mois d'intervalle l'Alliance Atlantique et le Conseil de l'Europe - dans lesquels il aura par la suite des fonctions éminentes. Parmi ces «repeat players» se trouvent les figures bien connues de Konrad Adenauer pour l'Allemagne, Paul Van Zeeland pour la Belgique, Robert Schuman pour la France, Carlo Sforza pour l'Italie ou

Field of Power”, International Political Sociology, 5-3, 2011, pp. 335-339 ; "Construction des espaces de pouvoir transnationaux en Europe », in Cohen A., Lacroix B. et Riutort P. (eds.), Nouveau manuel de science politique, Paris, La Découverte, 2009, pp. 611-624.

3. Cette démarche est longuement explicitée dans le numéro spécial : Bigo D., Madsen M. R. (eds.), "Bourdieu and the International”, International Political Sociology, 5-3, 2011.

4. L’Association Européenne de Libre Échange (AELE), créée par la Convention de Stockholm en 1960, et la Conférence, puis Organisation pour la Sécurité et la Coopération en Europe (OSCE) créée en 1975 par l'Acte final d'Helsinki, sont exclues du champ de cet article. 
Joseph Bech pour le Luxembourg. Mais il implique aussi que les jurisconsultes chargés de la rédaction des traités au sein des différents ministères nationaux passent bien souvent d'un texte à l'autre dans les mêmes conditions, à l'image de Paul Reuter dans le cas de la France, qui passe de la négociation du Traité CECA à la négociation du Traité CED qui se déroulent en parallèle en 195051. Tout un personnel politique et bureaucratique national se trouve ainsi investi du travail de définition juridique des institutions européennes, les sièges, les voix, les règles, les procédures, dont ce même personnel sera par la

Tableau 1 - Les organisations européennes et atlantiques (1947 / 1977)

\begin{tabular}{|c|c|c|c|c|c|c|c|}
\hline & 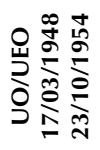 & 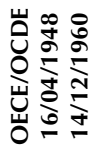 & 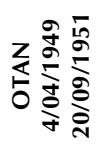 & 岁 & 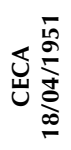 & 岂 & 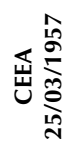 \\
\hline Allemagne & (?) & () & () & () & (:) & (:) & (:) \\
\hline Belgique & (:) & (;) & (;) & (;) & (:) & (;) & (:) \\
\hline France & (;) & (:) & (;) & (:) & (:) & (;) & (:) \\
\hline Italie & (:) & (:) & (:) & (:) & (:) & (;) & (:) \\
\hline Luxembourg & (:) & (:) & (:) & (;) & (:) & (:) & (:) \\
\hline Pays-Bas & (:) & (:) & (;) & (;) & (:) & (:) & (:) \\
\hline Royaume-Uni & (:) & (:) & (;) & (;) & () & () & (?) \\
\hline Danemark & & (;) & (:) & (;) & () & () & () \\
\hline Irlande & & (:) & & (:) & () & () & ( \\
\hline Grèce & & (;) & () & () & & & \\
\hline Islande & & (;) & (:) & (๑) & & & \\
\hline Norvège & & (:) & (:) & (:) & & & \\
\hline Portugal & & (:) & (:) & () & & & \\
\hline Turquie & & (;) & () & (9) & & & \\
\hline Autriche & & (;) & & (๑) & & & \\
\hline Suède & & (;) & & (:) & & & \\
\hline Suisse & & (;) & & (๑) & & & \\
\hline Espagne & & () & & (ن) & & & \\
\hline Chypre & & & & () & & & \\
\hline Finlande & & () & & & & & \\
\hline Malte & & & & () & & & \\
\hline États-Unis & & () & (:) & & & & \\
\hline Canada & & () & (:) & & & & \\
\hline
\end{tabular}

(-) Membre fondateur $\odot$ Adhésion postérieure

OECE : Ce n'est pas I'Allemagne en tant que telle qui signe la Convention du 16 avril 1948, mais les commandements militaires des zones occidentales d'occupation. La République Fédérale d'Allemagne souveraine en devient membre en 1949. Les ÉtatsUnis et le Canada deviennent membres associés de l'OECE en 1950. L'Espagne ne participe aux travaux de l'OECE qu'en 1955 et en devient membre en 1959. La Finlande devient membre de I'OCDE en 1969. Le Japon (1964), l'Australie (1971) et la Nouvelle-Zélande (1973) sont aussi membres de I'OCDE.

OTAN : La Grèce et la Turquie adhèrent en 1952, I'Allemagne en 1955.

CE : La Grèce et la Turquie deviennent membre en août 1949, I'Allemagne et I'Islande en 1951, I'Autriche en 1956, Chypre en 1961, la Suisse en 1962, Malte en 1965, le Portugal en 1976 et l'Espagne en 1977.

UEO : L'Allemagne devient membre de l'UEO à sa création en 1954. 
suite investi du travail de définition pratique. Tous les étages de la hiérarchie institutionnelle de la Haute Autorité ou de la Commission européenne sont en effet constitués de négociateurs des Traités de Paris ou de Rome, à l'image des plus éminents d'entre eux : Jean Monnet ou Walter Hallstein.

Plus encore, une fois ces organisations créees, ce sont bien souvent les mêmes ministres qui siègent dans les différents conseils des ministres qui se trouvent au cœur de chaque triangle ou rectangle institutionnel, suivant un calendrier de toute évidence ajusté, au fil du temps, pour que les ministres des pays membres de toutes les organisations puissent siéger d'affilée dans les différents conseils. En décembre 1959, par exemple, les conseils des ministres du CE, de l'OTAN, de la CECA, de la CEE et de la CEEA - quelles que soient les variations subtiles que comporte leur dénomination officielle, le Comité des ministres (CE), le Conseil de l'Atlantique nord (OTAN), le Conseil spécial des ministres (CECA) ou le Conseil des ministres (CEE et CEEA) - sont organisés sur une semaine, du 14 au 18, juste avant Noël, la session de la "Communauté atlantique " étant interposée entre les sessions de la "Communauté européenne »: les questions politiques du CE le lundi, les questions économiques de la CECA le mardi, les questions militaires de l'OTAN du mardi au jeudi, puis à nouveau les questions économiques de la CEE, mais aussi les questions nucléaires de la CEEA le vendredi. Les ministres des Affaires étrangères Heinrich Von Brentano (Allemagne), Pierre Wigny (Belgique), Maurice Couve de Murville (France), Giuseppe Pella (Italie), Eugène Schaus (Luxembourg) et Joseph Luns (Pays-Bas) siègent par conséquent de manière continue durant la semaine - Pella et Von Brentano étant, il est vrai, représentés au Comité des ministres du CE - tandis que les ministres des Affaires économiques siègent au Conseil spécial des ministres de la CECA le mardi ${ }^{5}$.

Cette structure intergouvernementale centrale et transversale est la clé de voûte de ce qui pourrait être décrit comme un "appareil » interétatique permanent et spécialisé mais différencié, pour reprendre les termes de Max Weber et Norbert Elias ${ }^{6}$, simultanément chargé de la gestion des monopoles militaires et économiques étatiques ${ }^{7}$. Pour autant que le processus d'institutionnalisation de cet « appareil » ait en effet conduit à une division du travail international entre les grandes organisations intergouvernementales européennes et entre des fonctions militaires et économiques, de jure, séparées, certaines institutions politiques en apparence cloisonnées sont, de facto, transversales

5. Je tiens à remercier Rémi Augustyniak-Berzin, Maxime Brun et Loïc Duchateau, étudiants du Master 2 Affaires publiques / Gouvernance européenne de l'Université Paris I Panthéon Sorbonne, pour le recueil de ces données.

6. Weber M., Économie et société, t. 1 (« Les catégories de la sociologie »), Paris, Presses Pocket, 1995 (1921), p. 97 ; Elias N., La dynamique de l'Occident, Paris, Presses Pocket, 1990 (1939), p. 26.

7. Cohen A. «Construction des espaces de pouvoir transnationaux en Europe », op.cit., pp. 617618. 
aux différents espaces de pouvoir militaires et économiques institués au cœur du champ du pouvoir européen. C’est en particulier le cas des institutions parlementaires européennes supranationales qui naissent dans le cours de ce processus - sur lesquelles porte plus particulièrement cet article.

Sans doute doit-on voir dans la genèse du « Parlement européen » 8 le produit des rivalités entre États pour faire triompher leur intérêt national respectif 9 . Sans doute doit-on aussi y voir le produit des rivalités entre élites aux capitaux distincts, les parlementaires ayant imposé une « assemblée » à un Jean Monnet originellement peu enclin ${ }^{10}$. Mais en aucun cas la naissance $d u$ «Parlement européen » ne peut être analysée en faisant abstraction du fait qu'il est, initialement, profondément enclavé dans un espace parlementaire qui comprend rapidement quatre « assemblées ». Cet espace parlementaire fragmenté n'a pas été anticipé comme tel et les parlementaires ne cesseront de vouloir le «défragmenter». Mais il constitue la matrice dont va émerger le «Parlement européen ». Pour autant, les travaux sur les assemblées extracommunautaires sont rarissimes 11 et, bien souvent, le produit de ces institutions elles-mêmes ${ }^{12}$. En outre, malgré une production abondante sur le Parlement européen ${ }^{13}$, les travaux scientifiques portant sur son institutionnalisation ${ }^{14}$ sont en réalité peu nombreux ${ }^{15}$ et n'échappent pas toujours à la célébration institutionnelle héroïque ${ }^{16}$.

\section{Un espace parlementaire transnational européen}

Au lendemain de la Seconde Guerre mondiale, les mobilisations transnationales en faveur d'une Europe unie se transforment rapidement en une lutte

8. On reviendra sur le sens de ces guillemets ci-dessous.

9. Sur ce point, voir les travaux de Berthold Rittberger : "Which Institutions for Post-War Europe? Explaining the Institutional Design of Europe's First Community”, Journal of European Public Policy, 8-5, 2001, pp. 673-708 ; “The Historical Origins of the EU’s System of Representation”, Journal of European Public Policy, 16-1, 2009, pp. 43-61.

10. Sur ce point : Cohen A., De Vichy à la Communauté européenne, Paris, PUF, 2012.

11. Voir par exception (et la revue de littérature) : Kraft-Kasack C., “Transnational Parliamentary Assemblies: A Remedy for the Democratic Deficit of International Governance”, West European Politics, 31-3, 2008, pp. 534-557.

12. Par exemple : Haller B., Une Assemblée au service de l'Europe. L'Assemblée parlementaire du Conseil de l'Europe 1949-1989, Strasbourg, Éditions du Conseil de l'Europe, 2006.

13. À titre indicatif : Abélès M., La Vie quotidienne au Parlement européen, Paris, Hachette, 1992 ; Costa O., Le Parlement européen, assemblée délibérante, Bruxelles, Éditions de l'Université de Bruxelles, 2001 ; Judge D., Earnshaw D., The European Parliament, Londres, Palgrave-Macmillan, 2008 (2e édition).

14. Par exemple sur le modèle classique de : Polsby N. W., “The Institutionalization of the U.S. House of Representatives”, The American Political Science Review, 62-1, 1968, pp. 144-168; ou Keohane R. O., "Institutionalization in the United Nations General Assembly", International Organization, 23-4, 1969, pp. 859-896 ; et voir : Lacroix B., Lagroye J. (eds.), Le Président de la République. Usages et genèses d'une institution, Paris, Presses de la FNSP, 1992.

15. Par exception : Rittberger B., Building Europe's Parliament. Democratic Representation Beyond the Nation-State, Oxford, Oxford University Press, 2005.

16. Mény Y. (ed.), La construction d'un parlement : 50 ans d'bistoire du Parlement européen, Luxembourg, Office des publications officielles des Communautés européennes, 2009. 
de pouvoir entre élites européennes (et américaines) opposant différentes stratégies pour reproduire au niveau supranational les formes institutionnelles les plus susceptibles de valoriser le type de capital dont chacune de ces élites dispose au niveau national ${ }^{17}$. Cette "mêlée » sans précédent se focalise en particulier sur la création d'une assemblée dont la définition concrète oppose les professionnels de la politique - qui doivent alors sans cesse réaffirmer que le pouvoir politique ne peut reposer que sur une représentation parlementaire, que ni le pouvoir militaire, ni le pouvoir bureaucratique, ni le pouvoir judiciaire ne sauraient être affranchis du contrôle politique, en un mot, que toute légitimité doit continuer de trouver sa source dans le suffrage universel, y compris au niveau supranational - aux élites économiques et bureaucratiques, mais aussi académiques ou militaires, qui défendent, de concert, bien que jouant des partitions différentes, des formes « nouvelles » de légitimité fondées sur des assemblées non parlementaires, des autorités non gouvernementales ou des juridictions non politiques.

Au rythme des succès et des échecs des différentes entreprises institutionnelles qui voient ainsi le jour dans cette dynamique de compétition entre élites, quatre assemblées supranationales sont alors créées, de la fin des années 1940 à la fin des années 1950, qui toutes rompent avec le principe de stricte égalité entre les États. Bien que cette révolution ait par la suite été minimisée, l'Assemblée consultative du Conseil de l'Europe introduit en effet une innovation majeure par rapport aux organisations intergouvernementales classiques, les sièges y étant distribués entre les États au suffrage universel indirect, sur la base (il est vrai très approximative) de leur population respective : 18 sièges pour la France, l'Italie, le Royaume-Uni et, à partir de 1951, l'Allemagne ; 7 sièges pour la Belgique, les Pays-Bas et, en août 1949, la Grèce ; 6 sièges pour la Suède, etc. [Tableau 2]. Cette représentation « proportionnée » des États va dès lors servir de « patron » à l'Assemblée commune de la CECA et, par la suite, à l'Assemblée des trois Communautés européennes, rebaptisée Assemblée parlementaire européenne en 1958, puis «Parlement européen » en 1962 18. Mais aussi aux deux « assemblées » non prévues par les traités constitutifs des organisations «dans le cadre » desquelles, ou plutôt "hors du cadre » desquelles elles ont été imposées par les parlementaires : l'Assemblée de l'UEO et la Conférence des parlementaires de l'OTAN, rebaptisée Assemblée de l'Atlantique nord en 1966. Comme le souligne en effet Paul Reuter dans la première édition de son manuel sur les organisations européennes, cette assemblée parlementaire officieuse, qui réunit « à titre

17. Cohen A., «De congrès en assemblées. La structuration de l'espace politique transnational européen au lendemain de la guerre », Politique européenne, 18, 2006, pp. 105-125.

18. Rappelons que ce sont les délégués de l'Assemblée "commune » aux trois Communautés européennes qui ont, de leur propre chef, rebaptisé « Assemblée parlementaire européenne » en 1958, puis «Parlement européen » en 1962 ce qui n'est alors qu’une « Assemblée »-dénomination qui ne sera officiellement consacrée par le droit des traités qu'en 1986 avec l'Acte unique. On n'utilisera donc le terme de «Parlement européen » que pour évoquer la période postérieure à 1979 . 
“privé" » des délégations des parlementaires des pays membres de l'OTAN, est un pur produit de la pratique : "N'étant pas prévue par les textes, elle a dû s'organiser dans une demi-clandestinité 19 ». À cela s'ajoute une brève, mais importante Assemblée «ad hoc » qui siège en parallèle des premières sessions de l'Assemblée commune de la CECA entre septembre 1952 et janvier 1953 aux fins de rédiger un projet de Constitution européenne - ou pour être plus exact un Traité portant statut de la Communauté européenne 20.

Au total, en 1957, à la veille de la signature des Traités de Rome, plus de 400 sièges de parlementaires sont donc à pourvoir - 429 pour être exact, en ne prenant pas en compte les sièges attribués aux représentants américains et canadiens dans la Conférence des parlementaires de l'OTAN. En 1977, à la veille de l'élection du « Parlement européen » au suffrage universel direct, le nombre total de sièges à pourvoir s'élève à près de $600-582$ suivant le même décompte ${ }^{21}$. Ces sièges sont distribués suivant des modalités qui sont la plupart du temps laissées à la libre appréciation des gouvernements et des parlements, voire des partis nationaux ${ }^{22}$. Ceci a d'ailleurs des effets notables sur la composition de ces assemblées. En 1957, pour s'en tenir au cas de l'Assemblée parlementaire européenne, la délégation allemande se compose exclusivement de membres du Bundestag 23, alors que les autres délégations se composent toutes de membres des deux chambres ${ }^{24}$, mais dans des proportions variables : aux deux tiers issus de l'Assemblée nationale et au tiers du Conseil de la République dans le cas de la délégation française ; aux trois cinquièmes de la Tweede kamer et au deux cinquièmes de la Eerste kamer der staten-generaal dans le cas de la délégation néerlandaise; et par moitié de la Chambre des représentants et du Sénat dans le cas de la délégation belge, et de la Camera dei deputati et du Senato della repubblica dans le cas de la délégation italienne. Le tout, suivant des règles de désignation elles aussi variables, à la proportionnelle des groupes parlementaires nationaux dans le cas des délégations allemande, belge et néerlandaise, au scrutin majoritaire dans le cas des délégations française, italienne et luxembourgeoise, pour une durée de mandat elle-même différente en fonction des délégations ${ }^{25}$.

19. Reuter P., Organisations européennes, Paris, PUF, 1965, pp. 137 et 145.

20. Cohen A., «La Constitution européenne. Ordre politique, utopie juridique et guerre froide ", Critique internationale, 26, 2005, pp. 119-131.

21. L'Acte portant élection des représentants de l'Assemblée au suffrage universel direct de 1976 représente le plus grand saut quantitatif dans l'évolution du nombre de sièges de ces assemblées, l'APE passant alors de 198 à 410 sièges sans élargissement.

22. Ginestet P., L'Assemblée parlementaire européenne, Paris, PUF, 1959 ; Stein E., “The European Parliamentary Assembly: Techniques of Emerging 'Political Control'", International Organization, 13-2, 1959, pp. 233-254.

23. Le Bundesrat n'ayant pas réussi à s'imposer face au Bundestag.

24. À l'exception de la délégation luxembourgeoise issue d'un parlement monocaméral.

25. Tout ceci démultipliant les chausse-trappes dans les décomptes qui vont suivre. 
Tableau 2 - Les enceintes parlementaires transnationales (1957 / 1967 / 1977)

\begin{tabular}{|c|c|c|c|c|c|c|c|c|c|c|c|c|c|c|c|c|c|c|c|c|}
\hline & $\begin{array}{l}\bar{\pi} \\
0 \\
\\
1 \\
0 \\
0 \\
0 \\
0\end{array}$ & 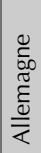 & 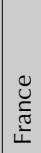 & $\stackrel{\frac{\varrho}{\underline{\pi}}}{ \pm}$ & $\mid \begin{array}{l}\frac{0}{\partial} \\
. \frac{0}{20} \\
\frac{D}{0} \\
\infty\end{array}$ & 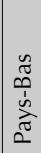 & 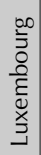 & 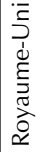 & 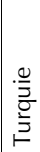 & 芯 & 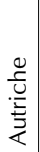 & $\begin{array}{l}\frac{0}{8} \\
\frac{d}{2} \\
\sim\end{array}$ & 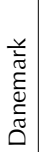 & 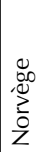 & 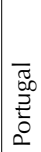 & 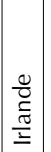 & $\begin{array}{l}\frac{d}{0} \\
\frac{\vec{T}}{\underline{\omega}}\end{array}$ & 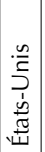 & 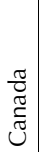 & $\frac{\pi}{0}$ \\
\hline $\begin{array}{c}\text { Conférence } \\
\text { parlementaire OTAN }\end{array}$ & 71 & 18 & 18 & 18 & 7 & 7 & 3 & 18 & 10 & 7 & & & 5 & 5 & 5 & & 5 & 36 & 12 & 174 \\
\hline Assemblée UEO & 71 & 18 & 18 & 18 & 7 & 7 & 3 & 18 & & & & & & & & & & & & 89 \\
\hline $\begin{array}{c}\text { Assemblée commune } \\
\text { CECA }\end{array}$ & 78 & 18 & 18 & 18 & 10 & 10 & 4 & & & & & & & & & & & & & 78 \\
\hline $\begin{array}{c}\text { Assemblée } \\
\text { consultative CE }\end{array}$ & 72 & 18 & 18 & 18 & 7 & 7 & 4 & 18 & 10 & 7 & 6 & 6 & 5 & 5 & & 4 & 3 & & & 136 \\
\hline
\end{tabular}

Nombre total de sièges disponibles dans chaque assemblée parlementaire supranationale européenne et nombre de sièges par pays en 1957

\begin{tabular}{|c|c|c|c|c|c|c|c|c|c|c|c|c|c|c|c|c|c|c|c|c|c|c|c|}
\hline & 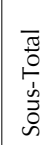 & 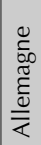 & 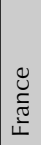 & $\stackrel{\varrho}{\cong}$ & $\frac{\frac{v}{2}}{\frac{0}{00}}$ & 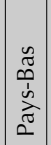 & 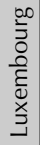 & 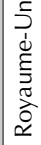 & $\begin{array}{l}\frac{0}{\frac{0}{3}} \\
\frac{0}{5} \\
\frac{1}{5}\end{array}$ & 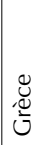 & 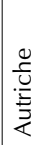 & $\left|\begin{array}{l}\frac{0}{0} \\
\frac{d}{D} \\
n\end{array}\right|$ & 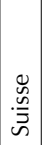 & 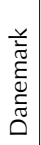 & $\begin{array}{l}0 \\
0 \\
0 \\
2 \\
0 \\
z \\
z\end{array}$ & $\begin{array}{l}\overline{\widetilde{0}} \\
\stackrel{0}{0} \\
\underline{\underline{0}} \\
0\end{array}$ & 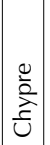 & 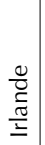 & $\frac{\stackrel{\Phi}{\pi}}{\frac{\pi}{z}}$ & $\begin{array}{l}\frac{0}{0} \\
\frac{\vec{\sigma}}{\underline{\underline{N}}}\end{array}$ & 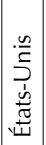 & $\begin{array}{l}\frac{\pi}{0} \\
\widetilde{\widetilde{E}} \\
\tilde{\mathbb{U}}\end{array}$ & $\stackrel{\bar{\pi}}{0}$ \\
\hline \begin{tabular}{|c|} 
Conférence \\
parlementaire OTAN
\end{tabular} & 71 & 18 & 18 & 18 & 7 & 7 & 3 & 18 & 10 & 7 & & & & 5 & 5 & 5 & & & & 5 & 36 & 12 & 174 \\
\hline Assemblée UEO & 71 & 18 & 18 & 18 & 7 & 7 & 3 & 18 & & & & & & & & & & & & & & & 89 \\
\hline $\begin{array}{l}\text { Assemblée parlemen- } \\
\text { taire CECA/CEE/CEEA }\end{array}$ & 142 & 36 & 36 & 36 & 14 & 14 & 6 & & & & & & & & & & & & & & & & 142 \\
\hline $\begin{array}{c}\text { Assemblée } \\
\text { consultative CE }\end{array}$ & 72 & 18 & 18 & 18 & 7 & 7 & 4 & 18 & 10 & 7 & 6 & 6 & 6 & 5 & 5 & & 4 & 4 & 4 & 3 & & & 150 \\
\hline
\end{tabular}

Nombre total de sièges disponibles dans chaque assemblée parlementaire supranationale européenne et nombre de sièges par pays en 1967

\begin{tabular}{|c|c|c|c|c|c|c|c|c|c|c|c|c|c|c|c|c|c|c|c|c|c|c|c|c|}
\hline & 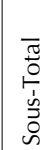 & 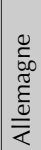 & 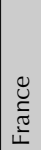 & $\stackrel{\cong}{ \pm}$ & 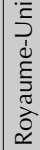 & $\mid \begin{array}{l}\frac{0}{\partial} \\
\frac{\partial}{00} \\
\frac{0}{0}\end{array}$ & 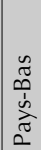 & 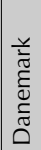 & $\begin{array}{l}\frac{0}{0} \\
\frac{\pi}{\underline{C}} \\
\text { I }\end{array}$ & 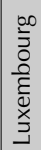 & 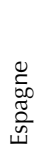 & $\begin{array}{l}\frac{0}{3} \\
\frac{0}{3} \\
\frac{3}{5}\end{array}$ & 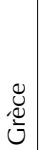 & $\begin{array}{l}\overline{\widetilde{\sigma}} \\
\mathrm{d} \\
\underline{0} \\
0 \\
0\end{array}$ & 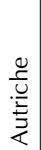 & $\frac{\mathbb{2}}{\mathrm{N}}$ & 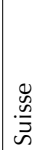 & $\begin{array}{l}0 \\
\infty \\
0 \\
2 \\
0 \\
Z\end{array}$ & 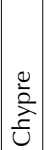 & $\frac{\mathscr{v}}{\frac{\pi}{2}}$ & $\begin{array}{l}\frac{0}{0} \\
\frac{\tilde{D}}{\underline{N}}\end{array}$ & 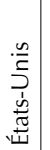 & $\begin{array}{l}\frac{\pi}{0} \\
\underset{\widetilde{J}}{\tilde{U}}\end{array}$ & $\bar{\pi}$ \\
\hline \begin{tabular}{|c|} 
Conférence \\
parlementaire OTAN
\end{tabular} & 94 & 18 & 18 & 18 & 18 & 7 & 7 & 5 & & 3 & & 10 & 7 & 5 & & & & 5 & & & 5 & 36 & 12 & 174 \\
\hline Assemblée UEO & 89 & 18 & 18 & 18 & 18 & 7 & 7 & & & 3 & & & & & & & & & & & & & & 89 \\
\hline $\begin{array}{l}\text { Assemblée parlemen- } \\
\text { taire CECA/CEE/CEEA }\end{array}$ & 198 & 36 & 36 & 36 & 36 & 14 & 14 & 10 & 10 & 6 & & & & & & & & & & & & & & 198 \\
\hline $\begin{array}{c}\text { Assemblée } \\
\text { consultative CE }\end{array}$ & 99 & 18 & 18 & 18 & 18 & 7 & 7 & 5 & 4 & 4 & 12 & 12 & 7 & 7 & 6 & 6 & 6 & 5 & 3 & 3 & 3 & & & 169 \\
\hline
\end{tabular}

Nombre total de sièges disponibles dans chaque assemblée parlementaire supranationale européenne et nombre de sièges par pays en 1977 


\section{Les interdépendances entre assemblées supranationales}

Dans une étude séminale, Ernst Haas soulignait que les délégués de l'Assemblée consultative du CE avaient rapidement compris leur rôle comme étant de contrôler les conseils des ministres y compris de l'OECE et de l'OTAN, dans le cadre de ce qu'il appelle un «inventaire européen » («European review ») ${ }^{26}$. Le cloisonnement institutionnel entre organisations intergouvernementales européennes est en effet rapidement contrebalancé par une série de facteurs juridiques et politiques, de sorte que cet espace parlementaire transnational est aussi un espace transparlementaire. De fait, en dépit des apparences, les quatre assemblées du CE, de la CECA-CEE-CEEA, de l'UEO et de l'OTAN sont fortement interdépendantes.

Les décomptes présentés dans ce qui suit sont issus de deux bases de données, sous forme de tableaux Excel, pour les années 1952 à 1956 et pour les années 1957 et 1958, 1967 et 1977 comprenant respectivement 340 et 660 individus (qui peuvent être les mêmes de I'une à l'autre). En leur état actuel, encore provisoire, sont renseignées des données minimales concernant le pays et l'assemblée d'origine pour les deux bases, les dates d'entrée et de sortie de l'Assemblée consultative du CE, de l'Assemblée commune de la CECA et de I'Assemblée " ad hoc " (ainsi que leur présence effective pour les années 1952, 1953 et 1954) dans la première de ces bases et, dans la deuxième de ces bases, la présence effective au sein de l'ACCE, de l'APE et de l'AUEO à trois dates d'intervalle en 1957 et 1958, 1967 et 1977, à quoi s'ajoutent quelques éléments sur la date de naissance, le type de diplôme, la profession et l'appartenance politique de ces parlementaires. Principales sources : 1 . Assemblée consultative du Conseil de l'Europe, Ordres du jour, Procès-verbaux : Quatrième session ordinaire, Strasbourg, Conseil de l'Europe, 1952 ; Ordres du jour, Procès-verbaux : Session extraordinaire, Strasbourg, Conseil de l'Europe, 1953 ; Ordres du jour, Procès-verbaux : Cinquième session ordinaire, Strasbourg, Conseil de l'Europe, 1953 ; Ordres du jour, Procès-verbaux : Sixième session ordinaire, Strasbourg, Conseil de l'Europe, $1954 ; 2$. Assemblée ad hoc, Informations et documents officiels de la commission constitutionnelle, Paris, Secrétariat de la commission constitutionnelle, 1952 ; Informations et documents officiels de la commission constitutionnelle, Paris, Secrétariat de la commission constitutionnelle, 1953 ; 3. Communauté européenne du charbon et de l'acier, Listes nominatives et renseignements généraux, s.l., Assemblée commune, 1953 ; Annuaire - Manuel de l'Assemblée commune, Luxembourg, Assemblée commune, Division des études et de la documentation, 1956 ; Annuaire - Manuel de l'Assemblée commune, Luxembourg, Assemblée commune, Division études, information et documentation, 1957 ; Parlement européen, Annuaire 1967, Luxembourg, Secrétariat général - Direction générale de la documentation parlementaire et de l'information, 1967 ; Annuaire des communautés européennes, Bruxelles, Éditions Delta, 1977 ; Assemblée de I'Union de l'Europe occidentale, Actes officiels : Troisième session, Strasbourg, Union de l'Europe occidentale, 1957 ; Actes officiels : Treizième session ordinaire, Paris, Union de l'Europe occidentale, 1967 ; Actes officiels : Vingt-troisième session ordinaire, Paris, Union de l'Europe occidentale, 1977 ; Parlement européen, Une assemblée en pleine évolution, Luxembourg, Office des publications officielles des Communautés européennes, 1989 (3e édition) ; http://assembly.coe.int/ ; http://www.ena.lu.

26. Haas E. B., Consensus Formation in the Council of Europe, Berkeley, University of California Press, 1960, p. 7. 
Une proportion importante de parlementaires siège en effet dans plusieurs de ces assemblées simultanément. Dans son Article 1er, le Protocole sur les relations avec le Conseil de l'Europe annexé au Traité CECA stipule en effet que : «Les gouvernements des États membres sont invités à recommander à leurs Parlements respectifs que les membres de l'Assemblée, qu'ils sont appelés à désigner, soient choisis de préférence parmi les représentants à l'Assemblée consultative du Conseil de l'Europe. » Ainsi, en 1952, au moment où se tient la séance constitutive de l'Assemblée commune de la CECA, le 10 septembre, suivie par la session régulière de l'Assemblée consultative du CE, le 15 septembre, le tout en parallèle avec la première session de l'Assemblée " ad hoc », 29 des 78 parlementaires membres de l'Assemblée commune de la CECA sont aussi membres de plein droit et siègent effectivement à l'Assemblée consultative du CE, 6 sont membres de plein droit de l'Assemblée consultative du CE mais se sont fait remplacer par leur suppléant (non membre de l'Assemblée commune de la CECA), 2 sont membres suppléants siégeant effectivement à l'Assemblée consultative du CE en remplacement de membres de plein droit (non membres de l'Assemblée commune de la CECA) et, finalement, 4 sont membres suppléants de l'Assemblée consultative du CE sans y siéger ${ }^{27}$. Bref, près de la moitié des parlementaires (37 sur 78 , soit $47,43 \%$ ) qui occupent un siège au sein de l'Assemblée commune de la CECA occupent aussi un siège de plein droit ou en tant que suppléant au sein de l'Assemblée consultative du CE à quelques jours d'intervalles - emportant au passage une surreprésentation des Français (11 individus) et des Italiens (10 individus) par rapport aux Allemands (5 individus). Quelques mois plus tard, en janvier 1953, en passant plus rapidement sur le détail 28,36 des 78 membres de l'Assemblée commune de la CECA sont aussi membres de l'Assemblée consultative du CE (46,15 \%) - avec la même surreprésentation des Français (11) et des Italiens (9) par rapport aux Allemands (5). Quant à l'Assemblée " ad hoc ", qui siège entre ces deux dates, elle comprend les 78 membres de l'Assemblée commune de la CECA, 16 observateurs et 8 membres cooptés. Parmi ces derniers, 21 sont alors membres de plein droit (14) ou membres suppléants (7) de l'Assemblée consultative du CE, dont 1 représentant du Danemark, de l'Irlande, de l'Islande et de la Norvège, 2 représentants de la Grèce, de la Suède et de la Turquie, et 3 représentants du Royaume-Uni aucun de ces pays n'étant, bien entendu, membres de la CECA - auxquels s'ajoutent 3 représentants de la France et de l'Italie et 2 représentants de l'Allemagne, ainsi que Christian Calmes, secrétaire du Conseil spécial des ministres, Max Kohnstamm, secrétaire de la Haute Autorité, et Jean-Claude Paris, secrétaire général du Conseil de l'Europe, tous trois cooptés ès qualité.

27. Ces comptages étant toujours sujets à caution, rendus particulièrement délicats du fait du système des suppléants spécifique à l'Assemblée consultative du CE.

28. Aux suppléés (4) et aux suppléants (1), doivent cette fois être ajoutés les excusés (11), qui ne semblent pas avoir été effectivement remplacés. 
Durant la phase initiale d'institutionnalisation de la CECA, il y a donc une très forte intrication institutionnelle et personnelle entre les trois assemblées, qui transforme alors Strasbourg, où elles siègent toutes, en un espace transparlementaire dans lequel les prises de position des uns ne peuvent être comprises que par rapport aux prises de position des autres.

Insensiblement, tout au long des années 1950, cette proportion de parlementaires multipositionnels va néanmoins décliner de manière linéaire, année après année, passant de près de la moitié à moins du quart du total des membres de l'Assemblée commune de la CECA : 29 sur 78 en 1954 (37,17\%), 23 sur 78 en 1955 (29,48 \%), 17 sur 78 en 1956 (21,79\%). En 1957, à la veille de l'entrée en vigueur des Traités de Rome, 19 des 78 membres de l'Assemblée commune de la CECA sont aussi membres de l'Assemblée consultative du CE, soit une proportion de 24,35\%. En 1958, au lendemain de l'entrée en vigueur des Traités de Rome, 27 des 142 membres de ce qui est entre temps devenu l'Assemblée parlementaire européenne sont aussi membres de l'Assemblée consultative du CE, soit une proportion de 19,01\% [Graphe 1]. Par rapport au total des sièges de l'Assemblée consultative du CE - soit 130 jusqu'à l'accession de l'Autriche en avril 1956 et 136 après cette date - cette proportion est bien entendu moindre, mais malgré tout significative : 27,69\% en $1953,22,30 \%$ en $1954,17,69 \%$ en $1955,12,50 \%$ en $1956,13,97 \%$ en 1957 et $19,85 \%$ en 1958 [Graphe 2]. Une dissociation entre les deux tendances due au fait que le nombre de sièges disponibles à l'Assemblée parlementaire européenne a brutalement augmenté en 1958, dépassant celui des sièges disponibles à l'Assemblée consultative du CE - ce qui sera à nouveau le cas en 1973.

\section{Graphe 1 - Évolution de la multipositionnalité parlementaire (1952-1958)}

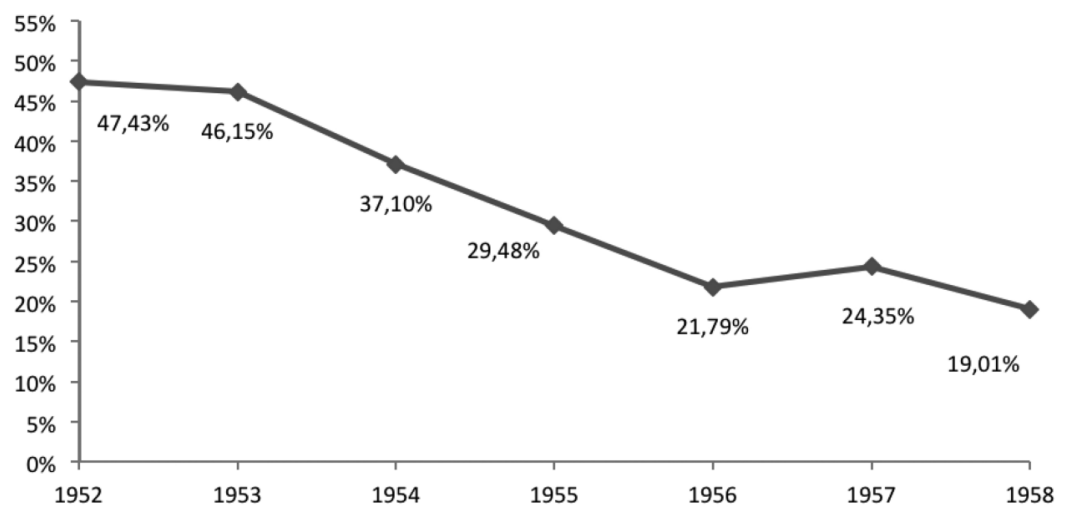

Membres de I'Assemblée commune de la Communauté européenne du charbon et de l'acier, puis de l'Assemblée parlementaire européenne, simultanément membres de I'Assemblée consultative du Conseil de l'Europe par rapport au total des sièges disponibles à I'Assemblée commune entre 1952 et 1958. 


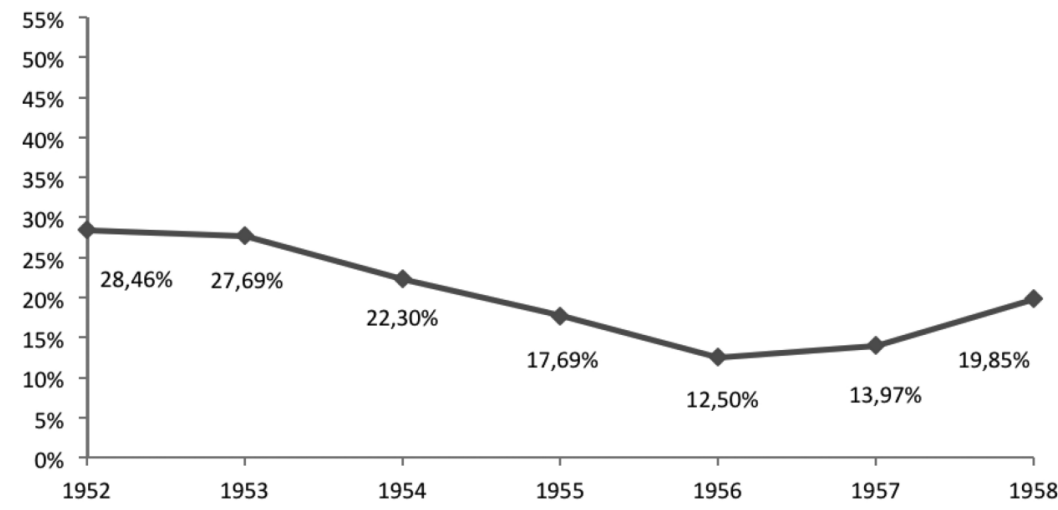

Membres de l'Assemblée consultative du Conseil de l'Europe simultanément membres de l'Assemblée commune de la Communauté européenne du charbon et de l'acier, puis de l'Assemblée parlementaire européenne par rapport au total des sièges disponibles à l'Assemblée consultative entre 1952 et 1958.

Enfin, par rapport au total des sièges de l'Assemblée consultative attribués aux États membres du CE et de la CECA, puis de la CECA-CEE-CEEA soit 72 sièges durant toute la période -, cette proportion est en réalité plus importante que par rapport au total des sièges de l'Assemblée commune, puis Assemblée parlementaire européenne : 51,38\% en 1952, 50,00\% en 1953 , $40,27 \%$ en $1954,31,94 \%$ en $1955,23,61 \%$ en $1956,26,38 \%$ en 1957 et $37,5 \%$ en 1958 [Graphe 3].

\section{Graphe 3 - Évolution de la multipositionnalité parlementaire (1952-1958)}

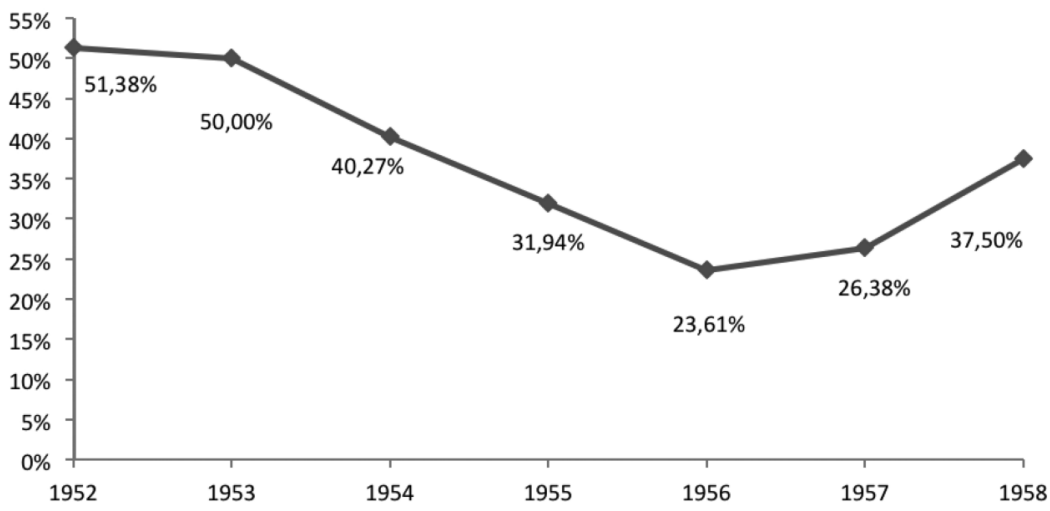

Membres de l'Assemblée consultative du Conseil de l'Europe simultanément membres de l'Assemblée commune de la Communauté européenne du charbon et de l'acier, puis de l'Assemblée parlementaire européenne, par rapport au total des sièges disponibles à l'Assemblée consultative pour les États membres du Conseil de l'Europe membres de la Communauté européenne du charbon et de l'acier, puis des Communautés européennes, entre 1952 et 1958. 
À partir de 1957, néanmoins, quel que soit le dénominateur retenu, on constate une accélération de ce processus de différenciation de l'Assemblée parlementaire européenne ${ }^{29}$. Ainsi, en 1967, seuls 3 des 142 membres de l'Assemblée parlementaire européenne sont aussi membres de l'Assemblée consultative du CE, soit une proportion de $2,11 \%$; et, en 1977, un nombre identique de 3 parlementaires membres de l'Assemblée parlementaire européenne sur un total qui est passé à 198 avec l'élargissement au Royaume-Uni, au Danemark et à l'Irlande, sont membres de ce qui, là aussi, est entre-temps (1974) devenu l'Assemblée parlementaire du CE, soit une proportion de $1,51 \%$ [Graphe 4].

\section{Graphe 4 - Évolution de la multipositionnalité parlementaire (1957-1967)}

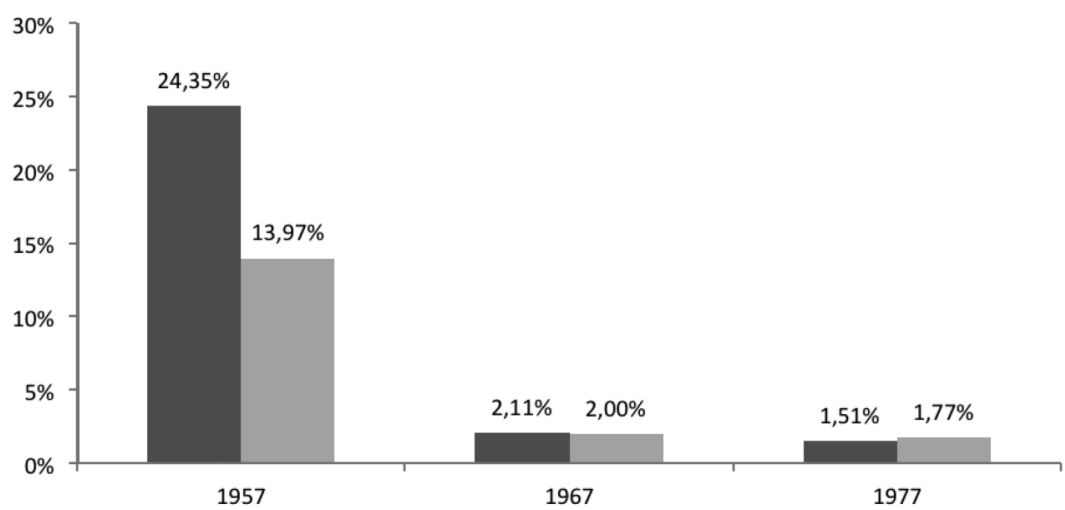

En gris foncé : Membres de l'Assemblée commune de la Communauté européenne du charbon et de l'acier, puis de l'Assemblée parlementaire européenne, simultanément membres de l'Assemblée consultative du Conseil de l'Europe, puis de l'Assemblée parlementaire du Conseil de l'Europe par rapport au total des sièges disponibles à l'Assemblée commune / parlementaire en 1957, 1967 et 1977.

En gris clair : Membres de I'Assemblée consultative du Conseil de l'Europe, puis de l'Assemblée parlementaire du Conseil de l'Europe, simultanément membres de l'Assemblée commune de la Communauté européenne du charbon et de l'acier, puis de l'Assemblée parlementaire européenne par rapport au total des sièges disponibles à l'Assemblée consultative / parlementaire en 1957, 1967 et 1977 .

Dans la durée, on assiste donc à une progressive dissociation des espaces parlementaires transnationaux et à une forte autonomisation du « Parlement européen » par rapport à l'Assemblée consultative, puis Assemblée parlementaire du CE, qui anticipe l'autonomisation subséquente de ce même « Parlement européen » par rapport aux parlements nationaux, avec l'élection au suffrage universel direct, qui supprime, de jure, le double mandat national

29. Au fond assez similaire au processus de différenciation de la Chambre des représentants américaine : établissement de frontières et élévation du coût d'entrée, division du travail et formalisation des rôles, concentration du capital et interdépendances accrues, etc. : Polsby N. W., op.cit. 
et européen (on se reportera sur ce point à l'article d'Ann-Christina L. Knudsen dans ce dossier), puis avec la dissociation, de facto, des mandats parlementaires européens et nationaux - la proportion de parlementaires détenant simultanément un mandat européen et un mandat national passant, au Parlement européen, de $31 \%$ en 1979 à moins de $7 \%$ en 199930.

Il faut dire que, dans le même temps, le nombre et la longueur des sessions ${ }^{31}$, ainsi que la charge de travail des délégués à l'Assemblée parlementaire européenne s'accroissent de manière considérable - malgré le caractère purement consultatif de ses avis ${ }^{32}$. En 1958, la production normative annuelle des institutions communautaires reste encore relativement faible : 13 règlements et aucune directive. Dès 1967, cette production s'élève à 309 règlements et 19 directives. En 1976, elle atteint 1287 règlements et 105 directives. Au total, une multiplication par plus de 100 fois la charge de travail par rapport à la première année de fonctionnement conjointe des trois Communautés européennes [Graphe 5]. De la même manière, le nombre des questions parlementaires s'accroît régulièrement, passant de 139 en 1964 à 2423 en 1979 (toutes questions écrites et orales confondues) [Graphe 6].

\section{Graphe 5 - Production normative des Communautés européennes (1958-1979)}

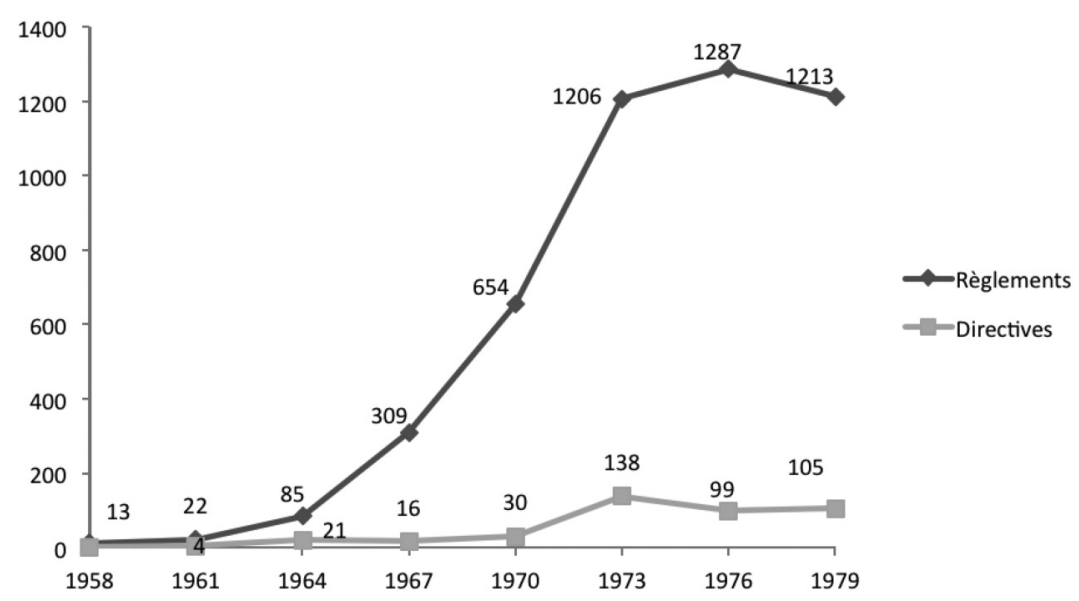

Règlements et directives adoptés annuellement entre 1958 et 1979 (intervalles de trois ans, année civile, incluant les rectificatifs). Source : Extraction EUR-Lex (11 avril 2012).

30. Beauvallet W., Michon S., « L’institutionnalisation inachevée du Parlement européen. Hétérogénéité nationale, spécialisation du recrutement et autonomisation ", Politix, 89, 2010, pp. 147-172, ici p. 158.

31. En 1957, le sénateur belge Roger Motz estimait à 77 le nombre de ses jours ouvrés à l'Assemblée commune et à l'Assemblée consultative : Stein E., op.cit., p. 240.

32. Sur ce point : Judge D., Earnshaw D., op.cit. 


\section{Graphe 6 - Questions parlementaires (1958-1979)}

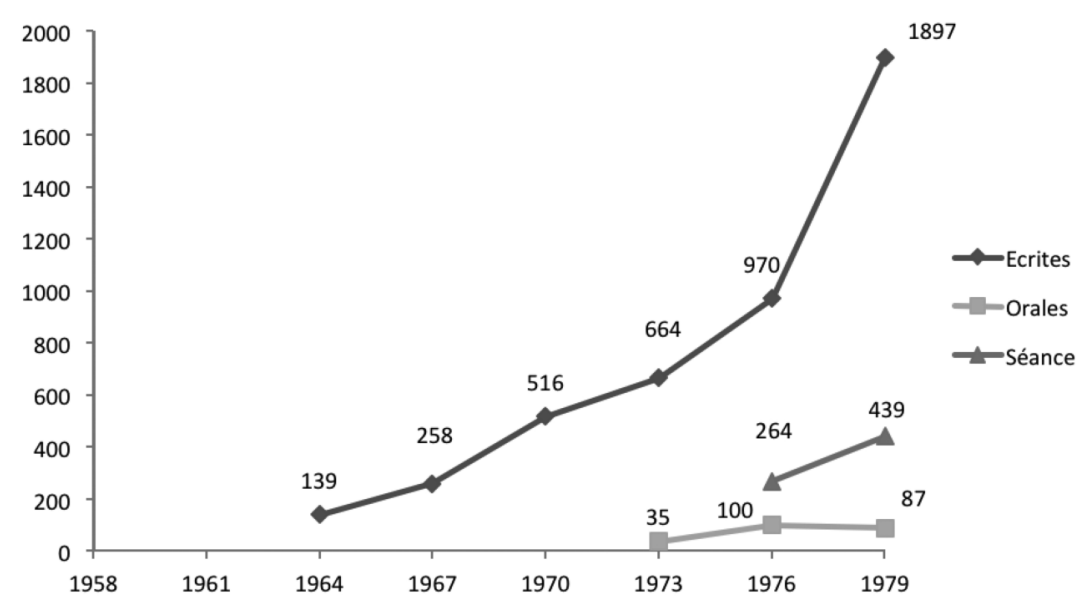

Questions écrites, questions orales et questions posées lors des séances de questions, annuellement, entre 1964 et 1979 (intervalles de trois ans, année civile, les questions parlementaires ne sont pas répertoriées dans EUR-Lex avant 1963). Source : Extraction EUR-Lex (11 avril 2012).

Ceci s'accompagne d'une augmentation exponentielle du budget des Communautés européennes. En 1958, le total des dépenses des Communautés européennes représente l'équivalent de 81,3 millions en unités de compte (UC). Dès 1970, ces dépenses atteignent un premier pic, soit l'équivalent de 3,5 milliards. En 1979, les dépenses des Communautés européennes s'élèvent à 14,7 milliards en unités de compte européennes (ECU) [Graphe 7].

\section{Graphe 7 - Dépenses budgétaires des Communautés européennes (1958-1979)}

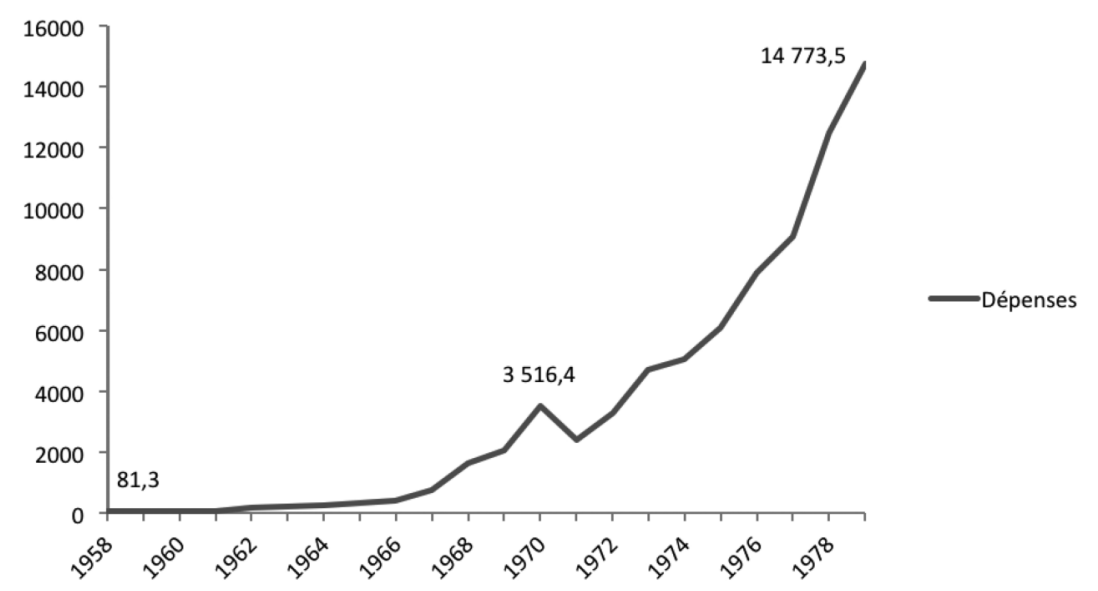

Dépenses annuelles des Communautés européennes exprimées en millions d'UC (unités de compte) jusqu'en 1977, puis en millions d'ECU (unités de compte européennes) jusqu'en 1979. Sont ici agrégés le total des dépenses du budget général de la CEE et des budgets spéciaux de la CECA, de la CEEA (incorporé au budget général en 1968) et du Fonds européen de développement (FED) entre 1958 et 1979. Source : Commission européenne, Budget de l'UE 2008. Rapport financier, Luxembourg, Office des publications de I'Union européenne, 2009, pp. 77-79 (Annexe II). 
Si ce processus de dissociation est très net entre les assemblées communautaire et extracommunautaires, les espaces parlementaires extracommunautaires restent, eux, fortement emboîtés. Deux nouvelles assemblées voient en effet le jour simultanément en 1955, l'Assemblée de l'UEO et la Conférence des parlementaires de l'OTAN. Or, leur personnel parlementaire est quasiment identique entre 1957 et 1977. Ainsi, en 1957, 86 des 89 membres de l'Assemblée de l'UEO sont aussi membres de l'Assemblée consultative du CE (96,62 \%) et, même si, en 1967, cette proportion décroît (72 sur 89, 80,89 \%), elle atteint $100 \%$ en 1977.

Ce processus s'accompagne paradoxalement d'une série d'initiatives de rationalisation en sens inverse pour organiser les relations entre ces différentes assemblées. Dès 1953, en conformité avec les Articles 2 et 3 du Protocole sur les relations avec le Conseil de l'Europe annexé au Traité CECA, qui prévoit que le rapport annuel de l'Assemblée commune et le rapport général de la Haute autorité de la CECA seraient communiqués à l'Assemblée consultative du CE ; et, plus encore, en vertu d'un " gentlemen's agreement » entre Jean Monnet, président de la Haute Autorité, et Lord Layton, premier vice-président de l'Assemblée consultative du CE, l'Assemblée commune de la CECA et l'Assemblée consultative du CE tiennent annuellement une réunion commune pour discuter le rapport de la Haute Autorité - pratique reconduite en 1958 et ceci jusqu'en 1979. Ce "droit d'inventaire commun" n'est jamais que la première d'une longue série d'initiatives allant dans le même sens. En 1956, le ministre britannique des Affaires étrangères Selwyn Loyd expose son «Grand Dessein » («Grand Design») devant la Conférence des parlementaires de l'OTAN en vue de remplacer les quatre assemblées existantes par une Assemblée parlementaire européenne unique ${ }^{33}$. En 1959, devant l'échec de cette proposition, le ministre belge des Affaires étrangères Pierre Wigny propose une redistribution plus pragmatique des pouvoirs entre ces assemblées, formulant au passage le vœu que l'Assemblée consultative du CE puisse contrôler le travail de l'OECE, qui ne comprend aucune assemblée parlementaire ${ }^{34}$. Suivies par plusieurs propositions du même type émanant de A. H. Robertson (1959), Arthur Conté (1961), Thorkil Kristensen (1962), Pierre Pflimlin (1963), John V. Lindsay (1963)...

33. Brumter C., The North Atlantic Assembly, Dordrecht, Martinus Nijhoff, 1986, p. 19 et suivantes sur lequel je m'appuie ici.

34. En avril 1959, une conférence organisée par Kenneth Lindsay, ancien parlementaire et ministre britannique, qui bénéficie alors du soutien de la Fondation Ford, peut ainsi s'appuyer sur un ensemble de « background papers » rédigés par des "spécialistes choisis avec soin », parmi lesquels une pléiade de politistes prestigieux (Hans Daalder, Jean Blondel, Mattei Dogan, U. W. Kitzinger, etc.), pour débattre de cette "rationalisation » : Lindsay K. (ed.), European Assemblies. The Experimental Period, 1949-1959, Londres, Stevens and sons, 1960 ; Les assemblées européennes (1949-1959). La période expérimentale, tiré à part ; et le compte rendu de Pierre Duclos dans la Revue française de science politique, 10-4, décembre 1960, pp. 974975. 
Mais l'initiative la plus spectaculaire en ce sens est sans doute celle qui a tout simplement conduit à la création d'une Assemblée "unique » pour les trois Communautés européennes. Au tout début du mois de février 1957, après une réunion commune des trois bureaux de l'Assemblée consultative du CE, de l'Assemblée commune de la CECA et de l'Assemblée de l'UEO, une délégation menée par Fernand Dehousse, Hans Furler et Georges Bohy est en effet reçue par les six ministres réunis en conférence pour la négociation des Traités de Rome au Val Duchesse à Bruxelles : «M. Dehousse, peut-on lire au procès-verbal, justifie ensuite la demande d'audience de la délégation par le fait qu'il était naturel que les Assemblées européennes soit consultées sur la constitution de la nouvelle Assemblée. D'une part, le caractère même des Assemblées justifie cette consultation et, d'autre part, la nature du problème traité fait que celui-ci aura nécessairement une incidence sur la structure et même sur l'existence des Assemblées actuellement en place. L'objectif à atteindre doit être une construction aussi rationnelle que possible et qui permette de progresser vers une unification de l'action parlementaire au moment où la "relance" devient une réalité. À plus longue échéance, il faut s'attacher à jeter les bases de ce qui pourrait être un jour une Assemblée européenne unique 35 ». Cette démarche aboutira au moins à un accord de principe pour éviter la création d'une nouvelle assemblée.

De fait, seules les initiatives de rationalisation institutionnelle internes aux Communautés européennes aboutiront. En 1957, la signature des Traités de Rome avait en effet entraîné une différenciation interne aux Communautés européennes avec la création de deux Commissions CEE et CEEA venant se juxtaposer à la Haute autorité CECA, mais aussi de deux Conseils des ministres distincts pour la CEE et la CEEA en plus du Conseil spécial des ministres de la CECA. (L'Assemblée parlementaire européenne étant la seule des institutions communautaires avec la Cour de Justice des Communautés Européennes (CJCE) - et, soit dit en passant, le service juridique de la Haute autorité puis des Commissions - à devenir immédiatement « commune » aux trois Communautés.) Avec la signature du Traité de fusion en 1965 et son entrée en vigueur en 1967, la Commission et le Conseil deviendront des institutions communes aux trois Communautés.

\section{Une élite du pouvoir parlementaire supranational}

Dans les années 1950, la «communauté européenne » reste un tout petit monde, au demeurant essentiellement masculin. Entre 1952 et 1956, 135 parlementaires occupent ainsi les 78 sièges de l'Assemblée commune de la CECA, dont 1 seule femme : la Néerlandaise Margaretha Klompé. Outre la relative homogénéité des attitudes envers la construction européenne résultant du

35. Parlement européen, Vers un parlement unique. L'influence de l'Assemblée commune de la CECA sur les Traités de Rome, Luxembourg, Parlement européen - Direction générale de la présidence, 2007, p. 145. 
processus de sélection (au vrai une « auto-sélection ») des membres des parlements nationaux ${ }^{36}$, deux caractéristiques se dégagent, qui dessinent les contours d'une petite élite du pouvoir parlementaire supranationale : la longévité et le capital juridique - deux traits qui ne manquent pas de perdurer bien au-delà de la période ${ }^{37}$.

Sur ces 135 parlementaires, 39 y siègent sans discontinuer entre l'automne 1952 et le printemps 1956 - dont Margaretha Klompé - avec des écarts importants entre les pays, puisque 10 de ces parlementaires sont Allemands et 2 sont Italiens, mais aussi entre les chambres, puisque les 10 parlementaires allemands sont tous issus du Bundestag, alors que seuls 2 parlementaires français sont issus de l'Assemblée nationale. On trouve là des syndicalistes, comme Willi Birkelbach, des professeurs, comme Antonio Boggiano Pico, des conseillers d'État, comme... Michel Debré, des journalistes, comme Jean Fohrmann, des industriels, comme Paul Kapteyn, des ingénieurs, comme Alain Poher - qui est le seul à avoir siégé sans discontinuer de 1952 à 1978, en particulier en tant que président de cette assemblée -, et bien sûr, des avocats, comme E. M. J. A. Sassen.

De fait, il est frappant de constater la prédominance des capitaux juridiques parmi le personnel de l'Assemblée parlementaire européenne et, plus encore, parmi la petite élite de parlementaires multipositionnels. En 1957, parmi les 78 parlementaires membres de l'Assemblée commune de la CECA, 34 sont en effet des « juristes » ${ }^{38}$, soit $43,58 \% 39$. Cette proportion restant étonnamment stable en 1967, puisque, parmi les 142 parlementaires membres de l'Assemblée parlementaire européenne, 60 sont des « juristes », soit un total

36. Kerr Jr. H. H., “Changing Attitudes Through International Participation: European Parliamentarians and Integration”, International Organization, 27-1, 1973, pp. 45-83. Au cœur de la théorie néo-fonctionnaliste se trouve en effet la question de savoir quel mécanisme de la sélection ou de la socialisation tend à déterminer le renforcement ou le changement des attitudes envers la construction européenne, que Haas constatait (ou prophétisait) dès 1958 : Haas E. H., The Uniting of Europe: Political, Social, and Economic Forces, 1950-1967, Stanford, Stanford University Press, 1958, p. 16.

37. Beauvallet W., Godmer L., Marrel G., Michon S., «La production de la légitimité institutionnelle au Parlement européen : le cas de la commission des affaires constitutionnelles », Politique européenne, 28, 2009, pp. 73-102 ; Marrel G., Payre R., " Des carrières au parlement. Longévité des eurodéputés et institutionnalisation de l'arène parlementaire ", Politique européenne, 18, 2006, pp. 69-104.

38. L'Annuaire Manuel de l'Assemblée commune de 1957 (op.cit.) et l'Annuaire du Parlement européen de 1967 (op.cit.) contiennent des notices biographiques plus ou moins précises pour l'ensemble du personnel des institutions communautaires, dont les parlementaires, tradition fort utile qui va se perdre par la suite, puisque l'Annuaire des Communautés européennes de 1977 n’en contient aucune. Ont ici été comptés comme « juristes » les individus pour lesquels il est fait mention d'un diplôme de doctorat en droit, même si leur activité professionnelle postérieure est autre que strictement juridique, ainsi que les individus qui ont effectivement exercé une profession juridique. Ce qui importe ici, plus que la définition nominale du capital juridique, c'est sa distribution relative en fonction de la multipositionnalité. On se reportera à l'article de Guillaume Sacriste dans ce dossier pour une analyse plus serrée de ce capital.

39. Pour des raisons qui tiennent au jeu des remplacements dans le courant de l'année 1957, aucune information n'a pu être recueillie pour 7 de ces 78 parlementaires $(n=71)$, de sorte que ce pourcentage pourrait s'élever à $47,88 \%$ au maximum. 
de $42,25 \%$ 40. Il y a là, en soi, un phénomène spécifique dans la mesure où les juristes sont alors en proportion déclinante, et en tout état de cause nettement inférieure dans les assemblées parlementaires nationales des différents pays membres des Communautés européennes ${ }^{41}$ - sans parler des effets de cette spécificité sur les débats parlementaires ${ }^{42}$. Mais, de manière plus frappante encore, cette proportion de juristes tend à s'accroître de manière très significative avec la multipositionnalité. En 1957, sur les 19 parlementaires membres de l'Assemblée commune de la CECA simultanément membres de l'Assemblée consultative du CE mentionnés plus haut, 12 sont aussi membres de l'Assemblée de l'UEO. Une petite minorité de 15,38\% des membres de l'Assemblée commune de la CECA sont donc simultanément membres de l'Assemblée consultative du CE et de l'Assemblée de l'UEO; mais une grosse majorité de 63,15\% de ces parlementaires doublement multipositionnels sont triplement multipositionnels. Dans le premier groupe de 19 parlementaires doublement multipositionnels, 14 sont des juristes, soit une proportion de 73,68 \%. Dans le second groupe de 12 parlementaires triplement multipositionnels, 10 sont des juristes, soit une proportion de 83,33\% [Graphe 8].

\section{Graphe 8 - Proportion de juristes parmi les parlementaires multipositionnels (1957)}

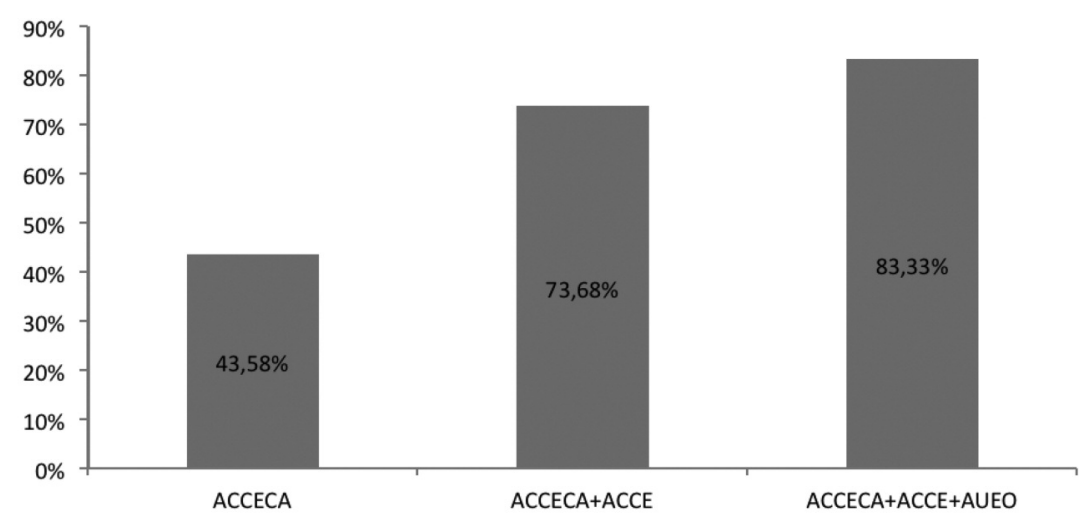

ACCECA : proportion de " juristes " parmi les membres de l'Assemblée commune de la Communauté européenne du charbon et de l'acier. ACCECA+ACCE : proportion de " juristes " parmi les membres de l'Assemblée commune simultanément membres de l'Assemblée consultative du Conseil de l'Europe. ACCECA+ACCE+AUEO : proportion de « juristes » parmi les membres de l'Assemblée commune membres de l'Assemblée consultative simultanément membres de l'Assemblée de l'Union de l'Europe occidentale en 1957.

40. De la même manière, 4 sièges sont vacants à la date choisie pour le décompte et 4 parlementaires n'ont pu être renseignés $(\mathrm{n}=134)$, de sorte que ce pourcentage pourrait s'élever à $44,77 \%$.

41. Best H., Cotta M., Parliamentary Representatives in Europe 1848-2000. Legislative Recruitment and Careers in Eleven European Countries, Oxford, Oxford University Press, 2000.

42. Sur ce point : Vauchez A., «Une élite d'intermédiaires. Naissance d'un capital juridique européen (1950-1970) ", Actes de la recherche en sciences sociales, 166-167, 2007, pp. 54-65. 
Ce processus de « sélection (si peu) naturel » ne manque pas de faire surgir certaines des figures centrales de la construction européenne par le droit. Il y a là un mélange « savant » de professionnels de la politique professionnels du droit : des professeurs de droit, comme Antonio Boggiano Pico, Fernand Dehousse, François de Menthon et Pierre-Henri Teitgen, des docteurs en droit, comme Jonkheer Van der Goes Van Naters et Hans Joachim Von Merkatz et des membres du Barreau, comme Kurt Georg Kiesinger, Hermann Kopf, Eugène Schaus et Paul Struye, ces titres et fonctions pouvant bien entendu se cumuler. Mieux. À la seule exception de Kiesinger, tous font partie du groupe des 39 députés à forte longévité. Au total donc, il y a une forte corrélation entre capital juridique, multipositionnalité et longévité.

Dans la longue durée, il est vrai, avec l'autonomisation de l'Assemblée parlementaire européenne, ce contingent de 12 parlementaires décroît drastiquement pour ne plus atteindre que 2 parlementaires en 1967, puis 1 parlementaire en 1977. Avec la multiplication du nombre de sièges européens offerts aux parlementaires nationaux et la dissociation des espaces parlementaires communautaire et extracommunautaires, plus généralement, on assiste à un accroissement mécanique du nombre de parlementaires nationaux « socialisés » à la construction européenne dans l'une et/ou l'autre de ces assemblées. À partir de 1958, 142 parlementaires ont désormais vocation à occuper un siège à l'Assemblée parlementaire européenne, ce qui est plus que le total des seuls 135 parlementaires ayant occupé un des 78 sièges de l'Assemblée commune de la CECA entre 1952 et $1956 .$. Pour autant, cette petite élite parlementaire ne perd pas prise.

\section{Conclusion}

Appréhender les organisations intergouvernementales européennes comme des espaces clairement distincts est particulièrement trompeur. Bien sûr, chacune de ces organisations a connu un rythme d'institutionnalisation endogène spécifique. Les groupes politiques, par exemple, officiellement reconnu en 1953 à l'Assemblée commune de la CECA (Article 33bis du règlement), ne sont créés qu'en 1956 à l'Assemblée consultative du CE (Article 41 du règlement). De la même manière, tandis que les membres de l'Assemblée parlementaire européenne décident unilatéralement de siéger par groupes politiques en 1958, dans ce que Pierre-Olivier Lapie appelle alors une "révolution des fauteuils » ${ }^{43}$, les membres de l'Assemblée consultative du CE vont continuer de siéger par ordre alphabétique... Cette institutionnalisation reste néanmoins partiellement impossible à comprendre sans analyser les interrelations entre chacune de ces assemblées, les groupes politiques, identiques dans

43. Cohen A., «La "Révolution des fauteuils" au Parlement européen. Groupes d’institution et institution du groupe », Scalpel. Cabiers de sociologie politique de Nanterre, 2-3, 1997, pp. 61 78. 
les deux assemblées, étant par exemple de puissants instruments de coordination des jeux.

Plus encore, cette structure transnationale d'institutions imbriquées permet de comprendre le pouvoir relatif que certains individus ont pu exercer sur la construction européenne dans son ensemble. Il n'existe virtuellement aucune position de pouvoir que Paul-Henri Spaak, par exemple, n'ait pas occupée de la fin des années 1940 au début des années 1960, de la présidence du Conseil de l'OECE (1948) à la présidence de l'Assemblée consultative du CE (1949-1951) et de la présidence de l'Assemblée commune de la CECA (1952-1954) au Secrétariat général de l'OTAN (1957-1961), sans oublier de mentionner le fait qu'il siège alors dans tous les conseils des ministres de ces organisations comme ministre belge des Affaires étrangères (1947-1949, 19541957, 1961-1966) et qu'il a aussi présidé le Mouvement européen (19501953)... Au vu de cette structure institutionnelle, par conséquent, il semble difficile d'opposer terme à terme le pouvoir intergouvernemental et l'entreprenariat supranational ${ }^{44}$, une élite de professionnels de la politique ayant joué une grande variété de rôles dans le champ du pouvoir européen naissant à travers les frontières institutionnelles et nationales.

44. Moravcsik A., “A New Statecraft? Supranational Entrepreneurs and International Cooperation”, International Organization, 53-2, 1999, pp. 267-306. 